...pharmaco-
logical
inhibition of
the integrated
stress
response
with ISRIB
can improve
cognitive
function
following TBI
in mice...

$\Rightarrow$

\title{
Integrated stress response linked to TBI
}

Integrated stress response inhibitor (ISRIB), a potent and selective small-molecule inhibitor of the integrated stress response, can ameliorate cognitive deficits in mouse models of traumatic brain injury (TBI), according to new research. The findings, reported by Susanna Rosi and colleagues, are the first to show that pharmacological inhibition of the integrated stress response with ISRIB can improve cognitive function following TBI in mice.

TBI, which is among the most common causes of death and disability in young adults, can cause cognitive deficits, including permanent memory impairment. In addition, patients with TBI have increased risk of developing Alzheimer disease and other neurodegenerative diseases.
Presently, no pharmacological treatments exist to treat patients with TBI; current therapeutic approaches comprise neurosurgical intervention or behavioural rehabilitation.

The integrated stress response is an adaptive pathway activated by eukaryotic cells in response to diverse stress stimuli to restore cellular homeostasis. The core regulatory step is the phosphorylation of the $\alpha$-subunit of the eukaryotic translation initiation factor 2 (eIF2 $\alpha$ ) by one of four eIF2 $\alpha$ kinases. Increased phosphorylation of eIF $2 \alpha$ in the brain prevents the formation of long-term memory. Previous studies have shown that TBI induces the phosphorylation of eIF2 $\alpha$. ISRIB, which can cross the blood-brain barrier, reverses the effects of eIF $2 \alpha$ phosphorylation.
Rosi and colleagues treated mice following focal TBI or concussive TBI with ISRIB, which they administered by intraperitoneal injection. The authors reported that treatment with ISRIB reversed the cognitive deficits in both mouse models, even when treatment was administered weeks after injury. These data suggest that the chronic cognitive deficits resulting from TBI might be treatable by targeting the integrated stress response pathway.

Alan Morris

ORIGINAL ARTICLE Chou, A. et al. Inhibition of the integrated stress response reverses cognitive deficits after traumatic brain injury. Proc. Natl Acad. Sci. USA http://dx.doi.org/10.1073/ pnas.1707661114 (2017) 